Herpertz-Dahlmann: Fluoxetin ist ab acht Jahren zugelassen, ich würde aber auch ein siebenjähriges Kind in einem individuellen Heilversuch behandeln, wenn es eine schwere Depression hat und sonst nichts hilft. Wie bei Jugendlichen geht auch bei vielen stationär aufgenommenen Kindern die Depression häufig innerhalb von drei Wochen ohne Arzneien zurück. Bei jungen Kindern spielen Umgebungsfaktoren eine große Rolle bei der Entstehung der Depression; deshalb hilft häufig ein Wechsel des Umfeldes.

Welche Hinweise findet man in der Literatur zum Nutzen von Antidepressiva speziell bei Kindern? Herpertz-Dahlmann: Eigentlich gibt es dazu kaum Untersuchungen. Ich kenne nur eine Studie, die ausschließlich Kinder untersucht hat. Eine Metaanalyse hat gezeigt, dass Kinder schlechter auf SSRI ansprechen als Adoleszente. Insgesamt haben wir aber viel zu wenige Daten, um zu sagen, ob die Wirksamkeit bei Kindern ähnlich gut ist wie bei Jugendlichen oder Erwachsenen.

Worauf sollte man bei einer ambulanten Therapie mit Antidepressiva achten?

Herpertz-Dahlmann: Man darf Kinder und Jugendliche nicht nur alle 14 Tage einbestellen. Man muss sich schon regelmäßig vergewissern: Haben sie suizidale Gedanken? Zeigen sie selbstverletzendes Verhalten? Beginnt man zudem mit sehr niedrigen Dosen, etwa mit $5 \mathrm{mg} / \mathrm{d}$ Fluoxetin, gibt es weniger Probleme mit Nebenwirkungen.
(2) Ab welchem Alter muss man erstmals mit Suizidgedanken rechnen?

Herpertz-Dahlmann: Schon bei einem acht- bis neunjährigem Kind können Suizidgedanken auftauchen. Dabei ist es sich nicht über die Konsequenz seines Handelns klar. Das Kind versteht nicht, dass sein Leben für „immer und ewig“ zu Ende ist, wenn es sich vor ein Auto stürzt. Kinder denken oft, der Tod ist ein Zustand, in dem sie abschalten und den Problemen entfliehen können. Der Begriff der Endgültigkeit ist in diesem Alter noch ganz schwierig zu fassen. Man muss also Suizidgedanken schon bei Achtjährigen absolut ernst nehmen!

Was lässt sich über die Prognose depressiver Kinder und Jugendlicher sagen?

Herpertz-Dahlmann: Sie ist nicht unbedingt gut. Ein beachtlicher Teil bleibt depressiv und hat auch im Erwachsenenalter ein erhöhtes Suizidrisiko. Sind Depressionen mit Störungen des Sozialverhaltens kombiniert, ist die Prognose besonders schlecht. Depressive Kinder haben ohne Frage ein erhöhtes Risiko, auch als Erwachsene Depressionen zu entwickeln. Man kann aber nicht sagen, wer als Kind depressiv ist, ist es automatisch auch später im Leben. Die Depression kann eine Episode sein, die vorbeigeht und nicht wieder kommt. Sie kann etwa als schwere Anpassungsstörung auf eine Scheidung der Eltern auftreten, und wieder verschwinden, wenn sich die Situation in der Familie beruhigt. Ganz wichtig ist jedoch eine frühe Behandlung, um die Chance auf Heilung zu verbessern.

Interview: Thomas Müller

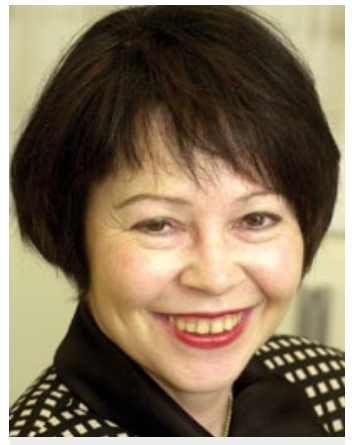

Professor Dr. med. Beate Herpertz-Dahlmann, Direktorin der Klinik für Kinder- und Jugendpsychiatrie, -psychosomatik und -psychotherapie am Universitätsklinikum Aachen

\title{
Freispruch für SSRI bei Minderjährigen?
}

Eine aktuelle Metaanalyse konnte keine Hinweise darauf finden, dass moderne Antidepressiva das Suizidrisiko bei Minderjährigen erhöhen. Im Gegensatz zu Erwachsenen senken die Medikamente das Risiko allerdings auch nicht.

Vor acht Jahren hatte die FDA eine Blackbox-Warnung für SSRI bei Minderjährigen herausgegeben. Grund waren Hinweise, dass unter diesen Antidepressiva das Suizidrisiko bei Kindern und Jugendlichen steigt. Laut einer damaligen Metaanalyse verdoppelte sich die Rate der Minderjährigen mit Suizidgedanken während einer sechsmonatigen SSRI-Therapie. Die Entscheidung der FDA stieß aber auch auf Kritik, so gab es Hinweise, dass bei einer längerfristigen SSRI-Therapie das Suizidrisiko auch bei Minderjährigen zurückgeht. In der aktuellen Arbeit wurden nun die Daten sämtlicher placebokontrollierter Studien zu Fluoxetin und Venlafaxin genauer geprüft. Dazu werteten die Autoren die in den Studien verwendeten Fragebögen aus, vor allem die Bereiche, die sich mit Suizidgedanken und suizidalem Verhalten wie Suizidversuchen auseinandersetzten.

Bei erwachsenen Depressiven nahmen Selbsttötungsgedanken und -absichten demnach deutlicher ab als mit Placebo, und zwar nach acht Wochen um $91 \%$ vs. $79 \%$. Bereits nach zwei Wochen zeigten sich Unterschiede zwischen Placebo und Verum. Die Suizidgedanken korrelierten dabei klar mit der Erkrankungsschwere: Je stärker die Depression zurückging, umso eher verschwanden auch die Suizidgedanken, und zwar sowohl in der Placebo- als auch in der Verumgruppe. Dies bestätigt Ergebnisse aus anderen Studien: Indem die Depression gelindert wird, sinkt auch das Suizidrisiko. Die Autoren berechneten, dass sich knapp $80 \%$ der antisuizidalen Wirkung von Fluoxetin und Venlafaxin auf ihre antidepressiven Eigenschaften zurückführen ließen. Anders sah es jedoch bei Minderjährigen unter Fluoxetin aus, dem einzigen bei Kindern und
Jugendlichen zugelassenen SSRI. Zwar gingen auch hier mit der Medikation die Depressionssymptome signifikant stärker zurück als mit Placebo, allerdings wirkte sich dies nicht entsprechend auf die Suizidgedanken aus. Nach acht Wochen ließ sich für die Placebogruppe ein Rückgang der Suizidgedanken und -handlungen um knapp über $60 \%$ berechnen, mit Fluoxetin nur um etwa $50 \%$, der Unterschied war aber nicht signifikant. Zwar äußerten auch hier die Patienten mit den schwersten Depressionen am häufigsten Suizidgedanken, anders als bei Erwachsenen verschwanden diese aber nicht im dem Maße, wie die Depressionssymptome zurückgingen. Suizidgedanken konnten also auch bei erfolgreicher Depressionstherapie persistieren. Immerhin, so die Autoren, nahmen sie unter der Medikation nicht zu. Dies stehe im Gegensatz zu den Befürchtungen der FDA..

mut

Gibbons RD et al. Arch Gen Psychiatry. Published online February 6, 2012 